\title{
Electrokinetic development length of electroviscous flow through a contraction
}

\author{
J. D. Berry ${ }^{1} \quad$ M. R. Davidson ${ }^{2} \quad$ D. J. E. Harvie ${ }^{3}$
}

(Received 30 January 2011; revised 26 September 2011)

\begin{abstract}
This study presents an analysis of the electrokinetic development length of electroviscous flow through a slit-like contraction. The effect of contraction ratio, electric double-layer thickness and wall surface charge on the electrokinetic development length of electroviscous flow is quantified. An order of magnitude expression for the electrokinetic development length is derived.
\end{abstract}

\section{Contents}

1 Introduction

C838

2 Method

C839

2.1 Model description . . . . . . . . . . . . . . . .

C839

http://journal . austms.org.au/ojs/index.php/ANZIAMJ/article/view/3923 gives this article, (c) Austral. Mathematical Soc. 2011. Published October 13, 2011. IssN 1446-8735. (Print two pages per sheet of paper.) Copies of this article must not be made otherwise available on the internet; instead link directly to this URL for this article. 
2.2 Development length definition .............. . C841

3 Results and discussion

C842

4 Conclusions

C849

A Derivation of excess charge $\Delta Q$

C850

\section{Introduction}

Micro-electro-mechanical systems (MEMS) offer great promise for many applications in biotechnology and chemical industries. Typical applications require the transport of fluids through non-uniform geometries including contractions and expansions. A fundamental understanding of flows at the micron scale is critical to ensure optimisation of MEMS. Electrokinetic effects due to the presence of ions in the fluid become important at these scales (Stone et al., 2004). The presence of a charged surface attracts counter-ions in the liquid, forming a diffuse electric double layer (EDL) (Masliyah and Bhattacharjee, 2006). Electroosmotic flow is driven by the application of an external electric field, whereas electroviscous flow is driven by applied pressures. Steady state electroviscous flow usually implies that the total current is zero. When the EDL thickness is significant compared to the channel width, the apparent viscosity of the fluid increases (Davidson and Harvie, 2007).

Modelling and analysis of flow through microfluidic devices generally assumes that fully developed flow is present. The development length of laminar flow in pipes and channels without electrokinetic effects has been well defined (Durst et al., 2005). The development length of electroosmotic flow in a microchannel has also been investigated (Yang et al., 2001, 2005). However, no attempt has been made to quantify the electrokinetic development length of electroviscous flow. Hence, this study presents numerical results and analysis in order to quantify the electrokinetic development length of electroviscous flow in a 


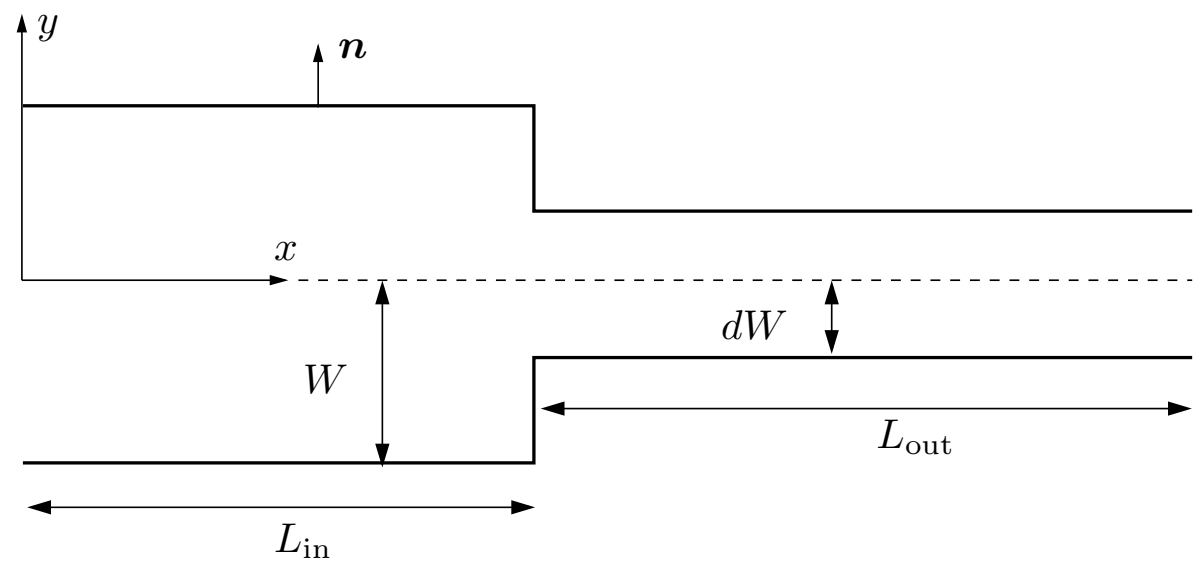

Figure 1: Schematic of contraction flow geometry with inlet channel halfwidth $W$ and contraction ratio $d$.

slit-like contraction.

\section{Method}

\subsection{Model description}

The model presented in this study consists of a $1: 1$ symmetric electrolyte solution flowing through a slit-like contraction (Figure 1). The inlet channel half-width is defined as $W$, and the outlet channel half-width as dW, where $d$ is the ratio of the contraction width to the inlet channel width. The fluid is assumed to have constant viscosity $\mu$, constant density $\rho$, permittivity $\varepsilon$, and mean velocity $\bar{V}$. The cations $\left(n_{+}\right)$and anions $\left(n_{-}\right)$present in the electrolyte have equal diffusivity coefficients $\mathrm{D}_{+}=\mathrm{D}_{-}=\mathrm{D}$, and equal valencies $z_{+}=-z_{-}=z$. The average ionic concentration throughout the fluid is $n_{0}$, representing the local geometric mean of both ion species (Harvie et al., 2011; Biscombe et al., 2011). A uniform, constant surface charge 
density $\sigma$ is assumed to be present on the walls of the channel.

The governing dimensionless equations are the Poisson equation relating the electric potential $U$ to the local charge density $\left(n_{+}-n_{-}\right)$,

$$
\nabla^{2} \mathrm{U}=-\frac{1}{2} \mathrm{~K}^{2}\left(\mathrm{n}_{+}-\mathrm{n}_{-}\right),
$$

the Nernst-Planck equation ensuring conservation of each ion species,

$$
\frac{\partial \mathfrak{n}_{ \pm}}{\partial \mathrm{t}}+\nabla \cdot\left(\mathbf{u} \mathbf{n}_{ \pm}\right)=\frac{1}{\operatorname{ReSc}}\left[\nabla^{2} \mathfrak{n}_{ \pm} \pm \nabla \cdot\left(\mathfrak{n}_{ \pm} \nabla \mathrm{u}\right)\right]
$$

and the Navier-Stokes equations with an electrical body force term,

$$
\begin{aligned}
& \frac{\partial \mathbf{u}}{\partial \mathrm{t}}+\nabla \cdot(\mathbf{u} \mathbf{u})=-\nabla \mathrm{P}+\frac{1}{\operatorname{Re}} \nabla \cdot\left[\nabla \mathbf{u}+(\nabla \mathbf{u})^{\top}\right]-\frac{\mathrm{BK}^{2}}{\mathrm{Re}^{2}}\left(\mathrm{n}_{+}-\mathrm{n}_{-}\right) \nabla \mathbf{u}, \\
& \nabla \cdot \mathbf{u}=0
\end{aligned}
$$

where $\mathbf{u}$ is the fluid velocity, and $\mathbf{P}$ is the pressure. Equations (1)-(4) have been non-dimensionalised using $\mathrm{W}$ as the length scale, $\overline{\mathrm{V}}$ as the velocity scale, $\mathrm{n}_{0}$ at the inlet as the ion number density scale, and $\mathrm{kT} / z e$ as the electric potential scale. The dimensionless numbers present in Equations (1)-(4) are

$$
\operatorname{Re}=\frac{\rho \overline{\mathrm{V} W}}{\mu}, \quad \mathrm{Sc}=\frac{\mu}{\rho \mathrm{D}}, \quad \mathrm{B}=\frac{\rho k^{2} \mathrm{~T}^{2} \varepsilon \varepsilon_{0}}{2 z^{2} e^{2} \mu^{2}}, \quad \mathrm{~K}^{2}=\frac{2 z^{2} e^{2} n_{0} W^{2}}{\varepsilon \varepsilon_{0} k T},
$$

where Re is the Reynolds number, Sc is the Schmidt number, $\mathrm{K}$ is the dimensionless inverse Debye length (proportional to the ratio of the channel half-width $W$ to the EDL thickness), and B is a material parameter that is fixed for a given liquid at a constant temperature. The constants $k, e$, and $\varepsilon_{0}$ are the Boltzmann's constant, elementary charge and the permittivity of free space, respectively.

The permittivity $\varepsilon$ of the wall is considered negligible, allowing the wall boundary condition for the electric potential to be written

$$
\frac{\partial U}{\partial n}=S=\frac{z e \sigma W}{\varepsilon \varepsilon_{0} k T}
$$


where $\boldsymbol{n}$ is the normal vector at the channel wall pointing out from the flow, and $\mathrm{S}$ is the dimensionless surface charge density.

The Reynolds number is set to 0.01 , the Schmidt number to 1000 , and the $\mathrm{B}$ parameter to $2.34 \times 10^{-4}$; these are typical values for water in a glass microchannel (Davidson and Harvie, 2007). A uniform staggered grid with 32 mesh cells per unit length $W$ is used to solve the governing equations given by Equations (1)-(4). Davidson and Harvie (2007) gave a detailed description of the numerical method. Simulations on a grid with 16 mesh cells per unit length $W$ yielded a difference of less than $1 \%$ for the overall electric potential drop.

The simulation is integrated forward in time until the total dimensionless current passing through both the inlet and outlet to the domain falls below $10^{-7}$, ensuring that the electroviscous flow solution has been reached.

\subsection{Development length definition}

To determine the development length of the electroviscous flow in the contraction, the following method was applied. First, the axial electric field profile $E_{x}$ at the outlet of the domain is compared to the axial electric field profile $E_{x}$ one channel half-width $W$ upstream. If the condition

$$
\left|\frac{E_{x}\left(x_{\text {out }}-W, y_{j}\right)-E_{x}\left(x_{\text {out }}, y_{j}\right)}{E_{x}\left(x_{\text {out }}, y_{j}\right)}\right|<10^{-4} \text { for all } y_{j}
$$

is met, the flow is considered fully developed. Here the subscript $j$ refers to the discrete values of $y$ defined on the computational mesh. The development length $L_{d}$ is then defined as the distance along the contraction where the condition

$$
\left|\frac{E_{x}\left(L_{d}, y_{j}\right)-E_{x}\left(x_{\text {out }}, y_{j}\right)}{E_{x}\left(x_{\text {out }}, y_{j}\right)}\right|<10^{-2} \text { for all } y_{j}
$$

is first met. This definition of development length is analogous to that for purely hydrodynamic flow, except that it is based on the axial electric 
a)

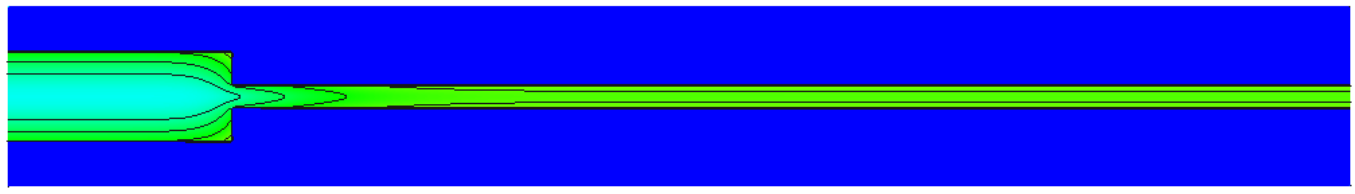

b)
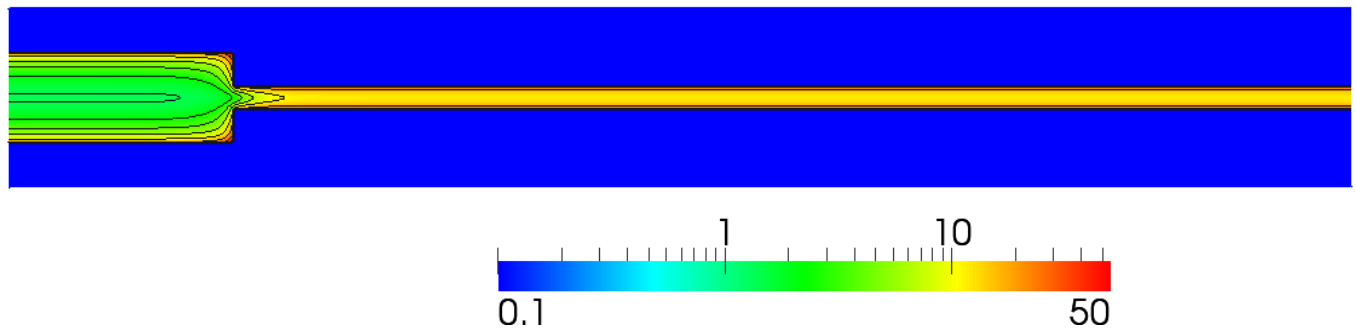

Figure 2: Magnitude of dimensionless charge density distribution $\left(\boldsymbol{n}_{+}-\boldsymbol{n}_{-}\right)$ for scaled inverse Debye length $\mathrm{K}=2$ and contraction ratio $\mathrm{d}=0.25$ : (a) surface charge density parameter $S=1$; (b) surface charge density parameter $S=4$. Flow is from left to right. The contour scale is logarithmic.

field rather than the velocity. The development length is this study is based on the axial electric field because in electroviscous flow with Peclet number $\mathrm{Pe}=\operatorname{Re} \mathrm{Sc}=10$ the velocity profile is relatively insensitive to the electroviscous resistance, whereas the generated axial electric field is not. Another suitable quantity is the local ion concentration $n_{0}$, not considered in this study.

\section{Results and discussion}

The magnitude of the dimensionless charge density distribution in the channel for scaled inverse Debye length $K=2$ and contraction ratio $d=0.25$ is shown in Figure 2. The contour scale is logarithmic to show clearly the regions 
a)

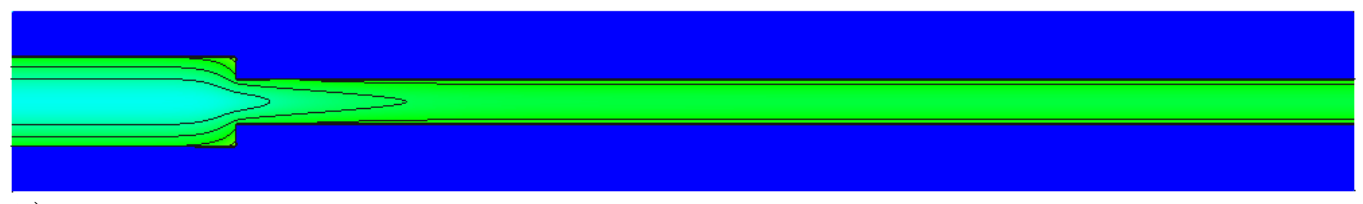

b)
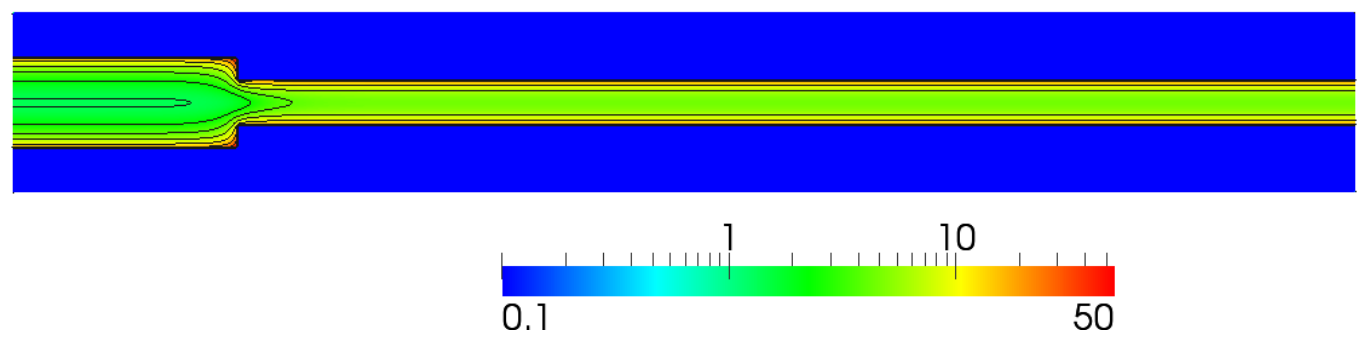

Figure 3: Magnitude of dimensionless charge density distribution $\left(\mathrm{n}_{+}-\right.$ $n_{-}$) for scaled inverse Debye length $\mathrm{K}=2$ and contraction ratio $d=0.5$ : (a) surface charge density parameter $S=1$; (b) surface charge density parameter $S=4$. Flow is from left to right. The contour scale is logarithmic.

of high and low charge densities present. As the flow adjusts from fully developed channel flow in the inlet to fully developed channel flow in the contraction, it is apparent that there is a region of undeveloped flow that starts just upstream of the contraction and extends downstream. The length of the undeveloped region is much longer for the lower surface charge density parameter depicted.

Figure 3 shows the magnitude of dimensionless charge density distribution in the channel for scaled inverse Debye length $\mathrm{K}=2$ and contraction ratio $\mathrm{d}=0.5$. Again, a region of undeveloped flow is observed, with the lower surface charge density $S=1$ having a longer undeveloped region.

To elucidate the effect of surface charge density $S$ on the development length, the axial electric field $E_{x}$ along the centreline of the channel is plotted in Figure 4a) for scaled inverse Debye length $K=2$ and contraction ratio 
$d=0.25$, and various values of $S$. For all values of surface charge density $S$ plotted, the axial electric field $E_{x}$ is constant in the inlet until a short distance upstream of the contraction. The axial electric field $E_{x}$ then increases in response to the change in geometry, eventually attaining a steady value at some distance downstream of the contraction. The fully developed axial electric field magnitude in the contraction decreases with surface charge density $S$. Despite this, the distance over which the axial electric field $E_{x}$ reaches the fully developed value in the contraction increases with decreasing surface charge density $S$.

Figure 4b) shows the axial electric field profile across the channel at various distances downstream of the contraction, for scaled inverse Debye length $\mathrm{K}=2$, contraction ratio $\mathrm{d}=0.25$ and surface charge density parameter $S=2$. Immediately after the contraction, at $x=5$, the axial electric field profile is non-uniform, and much lower than the fully developed profile. However, further downstream the axial electric field profile becomes uniform as it approaches the fully developed profile.

The development length of the flow based on the axial electric field profile is depicted in Figure 5 for two values of scaled inverse Debye length $\mathrm{K}$ and contraction ratio $\mathrm{d}$. The development length of the flow increases with decreasing $S$ for all values of $K$ and $d$. This increase is most noticeable at the lower scaled inverse Debye length $K=2$. At low values of dimensionless surface charge densities $S$ the development length is greater for lower contraction ratios. This effect is reversed as $\mathbf{S}$ increases.

To quantify the effect of scaled inverse Debye length K, dimensionless surface charge density $S$ and contraction ratio $d$ on the development length, an expression is derived for the excess charge $\Delta \mathrm{Q}$ present in the channel due to the contraction. Because the channel changes in width as the liquid flows through the contraction, the fully developed axial electric field magnitude increases. Applying Gauss' theorem to Equation (1), a difference in average axial electric field magnitude must result in an accumulation of charge at and around the contraction. This charge accumulation is equal to the total 

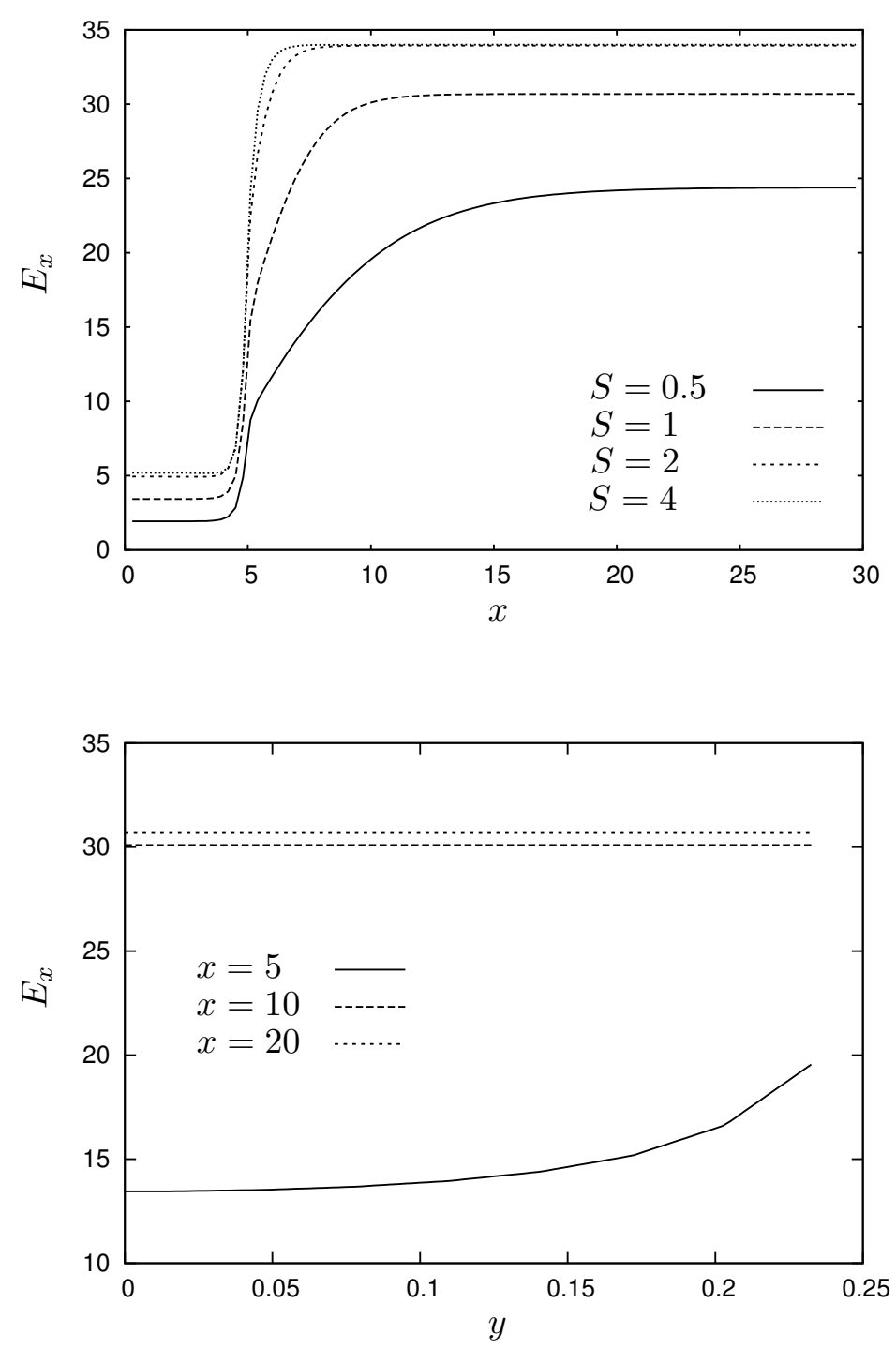

Figure 4: (a) Dimensionless axial electric field $E_{x}$ on the centreline for scaled inverse Debye length $\mathrm{K}=2$, contraction ratio $\mathrm{d}=0.25$ and different values of surface charge density parameter $S$; (b) dimensionless axial electric field $E_{x}$ across the contraction for scaled inverse Debye length $\mathrm{K}=2$, contraction ratio $\mathrm{d}=0.25$ and surface charge density parameter $\mathrm{S}=2$ for various distances downstream of the contraction. The contraction begins at $x=5$. 


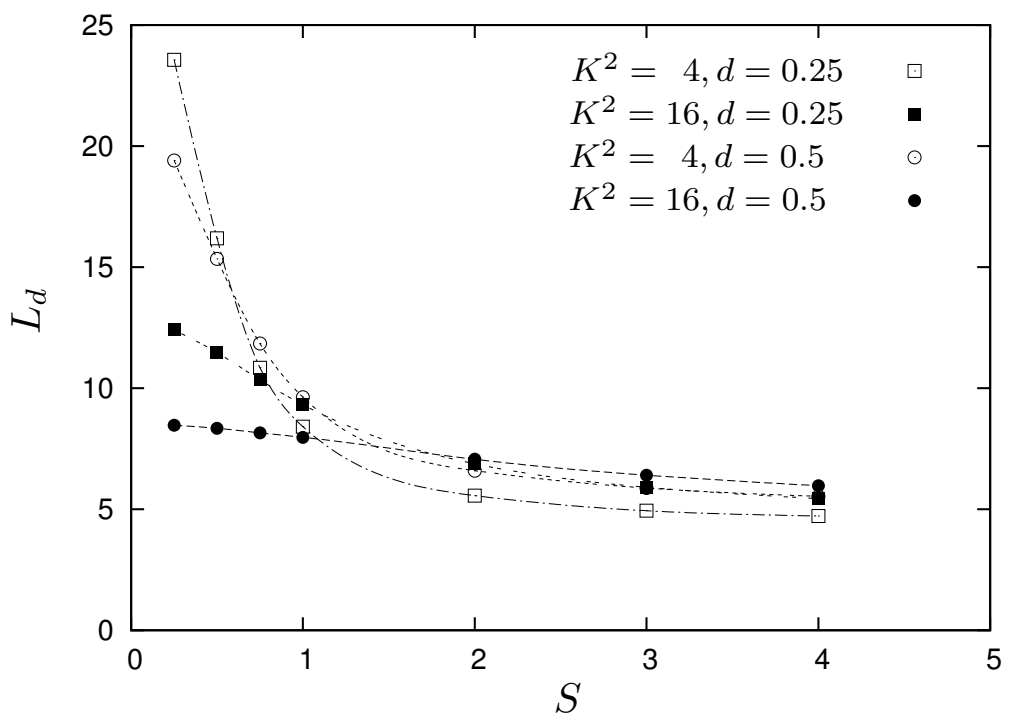

Figure 5: Development length $\mathrm{L}_{d}$ of the axial electric field profile as a function of dimensionless surface charge density $S$ for different scaled inverse Debye lengths $\mathrm{K}$ and contraction ratios d. Best fit curves are also shown.

charge in the channel over a suitable control volume beginning upstream and ending downstream of the contraction, minus the charge present on the channel wall parallel to the flow over the length of the control volume. The resultant quantity is the excess charge $\Delta \mathrm{Q}$ (Appendix A), where

$$
\Delta \mathrm{Q}=\frac{4}{\mathrm{~K}^{2}}\left[\mathrm{dE}_{\text {out }}-\mathrm{E}_{\mathrm{in}}-\mathrm{S}(1-\mathrm{d})\right]
$$

in which $E_{\text {in }}$ and $E_{\text {out }}$ refer to the fully developed axial electric fields in the inlet and contraction respectively. The fully developed axial electric fields are also functions of $\mathbf{K}$ and $\mathbf{S}$ (Harvie et al., 2011; Biscombe et al., 2011). Figure 6 shows the excess charge present in the channel calculated numerically and analytically with Equation (9). The numerical and analytical results are consistent.

It is reasonable to assume that the development length of the flow is pro- 


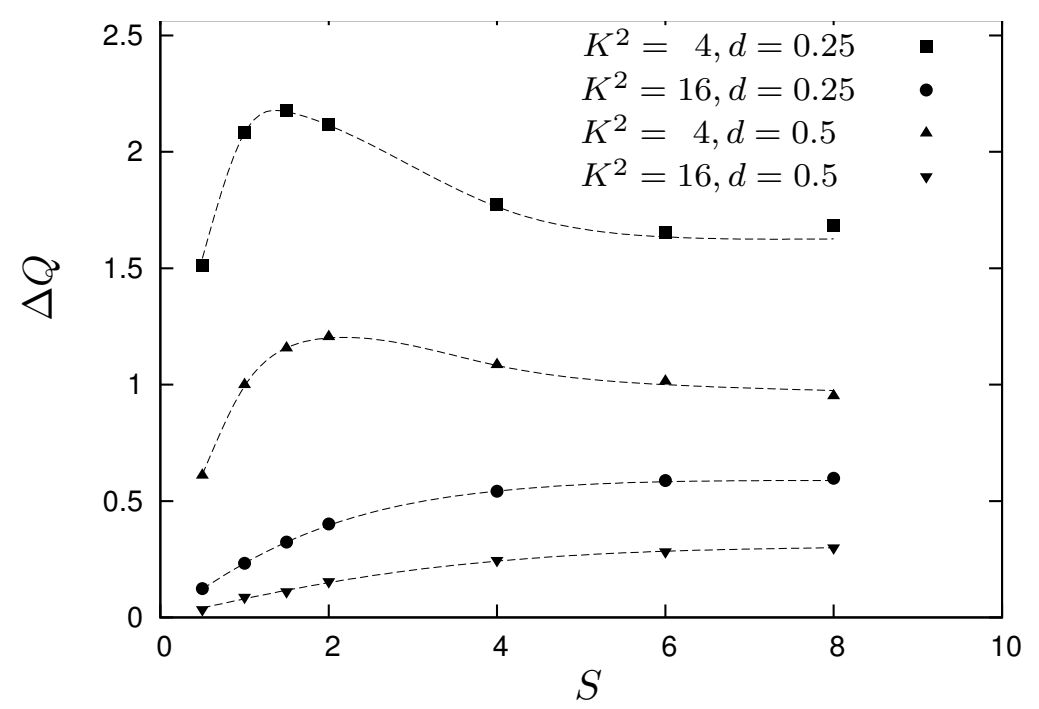

Figure 6: Excess charge $\Delta \mathrm{Q}$ in channel due to the contraction for different scaled inverse Debye lengths $\mathrm{K}$ and contraction ratios d: comparison of numerical results (symbols) and Equation (9) (lines).

portional to the amount of excess charge present due to the contraction. If the magnitude of the excess charge is large, then the flow will take longer to recover to full development. Hence, an order of magnitude relationship for the development length $\mathrm{L}_{\mathrm{d}}$ is obtained by dividing the total excess charge $\Delta \mathrm{Q}$ by the magnitude of the charge $\mathbf{q}$ across the cross-section of fully developed flow in a uniform channel of half-width $W$ :

$$
|\mathrm{q}|=\frac{4 \mathrm{~S}}{\mathrm{~K}^{2}} \text {. }
$$

This gives an expression for the development length $L_{d}$ of the form

$$
\mathrm{L}_{\mathrm{d}} \propto \frac{\Delta \mathrm{Q}}{|\mathrm{q}|}=\frac{1}{\mathrm{~S}}\left[\mathrm{dE}_{\text {out }}-\mathrm{E}_{\text {in }}\right]-(1-\mathrm{d}) .
$$

This relationship is plotted in Figure 7 for several values of scaled inverse Debye length $\mathrm{K}$, contraction ratio $\mathrm{d}$ and dimensionless surface charge density $\mathrm{S}$. 


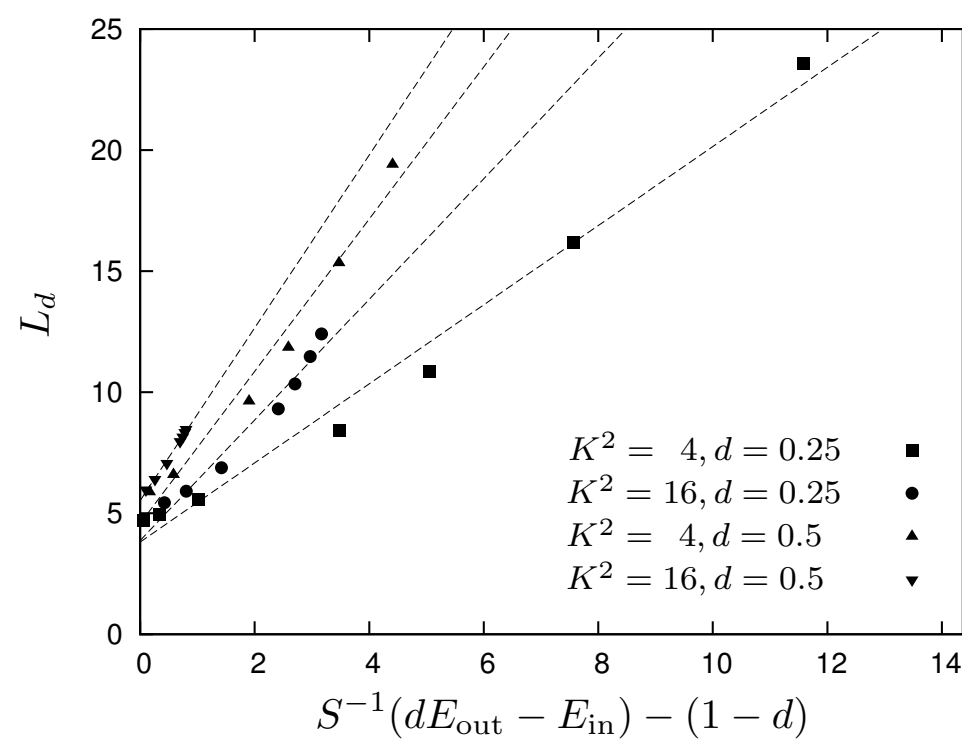

Figure 7: Development length $L_{d}$ based on axial electric field $E_{x}$ as a function of $S^{-1}\left(d E_{\text {out }}-E_{\text {in }}\right)-(1-d)$ for scaled inverse Debye lengths $K=2$ and 4 , contraction ratios $\mathrm{d}=0.25$ and 0.5 , and dimensionless surface charge densities $0.25 \leqslant S \leqslant 4$. The numerical results are shown by the symbols, and the lines of best fit (12) are plotted with dashed lines.

Also shown on the plot are the lines of best fit

$$
L_{d}=A\left[\frac{1}{S}\left(d E_{\text {out }}-E_{\text {in }}\right)-(1-d)\right]+B .
$$

The values of $A$ and $B$ are shown in Table 1. It is apparent that the development length increases monotonically with the function defined in Equation (11), showing that the function defined is a good indicator of development length magnitude. However, the assumption made in deriving Equation (11), defined in Equation (10), does not cause the data to collapse to a single curve, and a dependence on both contraction ratio $d$ and scaled inverse Debye length $\mathrm{K}$ is still present. Despite this, the gradients of the lines of best fit only 
Table 1: Best fit parameters for Equation (12)

\begin{tabular}{llll}
\hline $\mathrm{K}$ & $\mathrm{d}$ & $\mathrm{A}$ & $\mathrm{B}$ \\
\hline 2 & 0.25 & 1.6 & 3.8 \\
2 & 0.5 & 2.5 & 3.9 \\
4 & 0.25 & 3.1 & 4.6 \\
4 & 0.5 & 3.6 & 5.5 \\
\hline
\end{tabular}

vary between 1.6 and 3.6, demonstrating that Equation (11) is an accurate order of magnitude relationship for the electroviscous development length.

\section{Conclusions}

The electrokinetic development length of electroviscous flow in a slit-like contraction is quantified for different values of the scaled inverse Debye length $\mathrm{K}$, contraction ratio $\mathrm{d}$, and dimensionless surface charge density $\mathrm{S}$. Over the range of dimensionless surface charge densities $S$ studied, the development length $L_{d}$ increases with decreasing $S$ and scaled inverse Debye length $K$. For surface charge densities $S \gtrsim 1$, the development length increases with increasing contraction ratio $d$. To explain these observations, an analytical expression for the excess charge in the contraction is derived to obtain an expression for the development length. It is shown that the development length increases monotonically with the quantity $S^{-1}\left[\mathrm{dE}_{\text {out }}-E_{\text {in }}\right]-(1-d)$. An order of magnitude expression for the development length is

$$
\mathrm{L}_{\mathrm{d}} \sim 2\left[\frac{1}{\mathrm{~S}}\left(\mathrm{dE}_{\text {out }}-\mathrm{E}_{\text {in }}\right)-(1-\mathrm{d})\right]
$$

Future work will focus on refining the analysis for flows in square channels and pipes. 


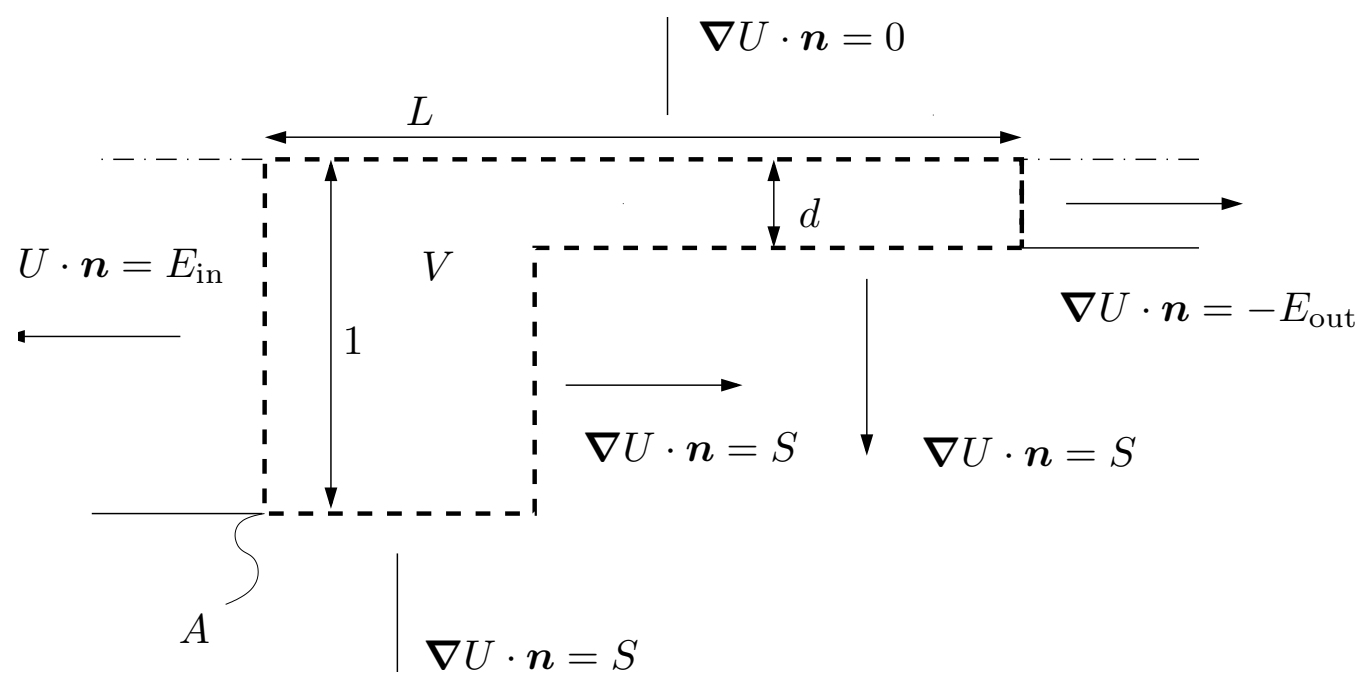

Figure 8: Control volume V (defined by the dashed line) of length L encompassing contraction.

\section{A Derivation of excess charge $\Delta Q$}

Consider the control volume $V$ depicted in Figure 8. The Poisson equation (1) is integrated over the control volume $\mathrm{V}$ to give

$$
\int_{V} \nabla^{2} U d V=-\frac{1}{2} K^{2} \int_{V}\left(n_{+}-n_{-}\right) d V .
$$

Gauss' divergence theorem reduces the left-hand side volume integral to a surface integral over the boundary of $\mathrm{V}$, yielding

$$
\int_{A} \nabla \mathrm{U} \cdot \mathrm{nd} A=-\frac{1}{2} \mathrm{~K}^{2} \int_{V}\left(\mathrm{n}_{+}-\mathrm{n}_{-}\right) \mathrm{dV} .
$$

If the length of the control volume $\mathrm{V}$ is long enough, the flow at either end is considered fully developed. Hence, Equation (15) is written as

$$
-\mathrm{dE}_{\text {out }}+\mathrm{E}_{\mathrm{in}}+\mathrm{SL}+\mathrm{S}(1-\mathrm{d})=-\frac{1}{2} \mathrm{~K}^{2} \int_{V}\left(n_{+}-n_{-}\right) \mathrm{dV},
$$


where $E_{\text {in }}$ and $E_{\text {out }}$ refer to the fully developed axial electric fields in the inlet and contraction respectively. The fully developed axial electric fields are functions of $\mathrm{K}$ and $\mathrm{S}$.

The integral on the right-hand side can be written as the addition of two contributions to the charge in the control volume

$$
-\frac{1}{2} K^{2} \int_{V}\left(n_{+}-n_{-}\right) d V=S L-\frac{1}{2} K^{2}\left[\frac{1}{2} \Delta Q\right],
$$

where the first term on the right-hand side of Equation (17) is the total charge on the channel wall parallel to the flow over the length of the control volume $\mathrm{L}$, and $\Delta \mathrm{Q}$ is the excess charge present in the channel due to the contraction. Substituting Equation (17) into Equation (16) yields

$$
-\mathrm{dE}_{\text {out }}+\mathrm{E}_{\mathrm{in}}+\mathrm{SL}+\mathrm{S}(1-\mathrm{d})=\mathrm{SL}-\frac{1}{4} \mathrm{~K}^{2} \Delta \mathrm{Q} .
$$

Rearranging to solve for $\Delta \mathrm{Q}$ gives

$$
\Delta \mathrm{Q}=\frac{4}{\mathrm{~K}^{2}}\left[\mathrm{dE}_{\text {out }}-\mathrm{E}_{\mathrm{in}}-\mathrm{S}(1-\mathrm{d})\right] .
$$

\section{References}

C. J. C. Biscombe, M. R. Davidson, and D. J. E. Harvie. Microfluidic circuit analysis II: Implications of ion conservation in series circuits. Journal of Colloid and Interface Science, 2011. doi:10.1016/j.jcis.2011.07.078. C839, C846

M. Davidson and D. Harvie. Electroviscous effects in low Reynolds number liquid flow through a slit-like microfluidic contraction. Chemical Engineering Science, 62(16):4229-4240, August 2007. doi:10.1016/j.ces.2007.05.006 C838, C841 
F. Durst, S. Ray, B. Ünsal, and O. A. Bayoumi. The development lengths of laminar pipe and channel flows. Journal of Fluids Engineering, 127(6): 1154, 2005. doi:10.1115/1.2063088 C838

D. J. E. Harvie, C. J. C. Biscombe, and M. R. Davidson. Microfluidic circuit analysis I: Ion current relationships for thin slits and pipes. Journal of Colloid and Interface Science, 2011. doi:10.1016/j.jcis.2011.07.076. C839, C846

J. H. Masliyah and S. Bhattacharjee. Electrokinetic and Colloid Transport Phenomena. Wiley-Interscience, Hoboken, New Jersey, 2006. C838

H.A. Stone, A.D. Stroock, and A. Ajdari. Engineering Flows in Small Devices. Annual Review of Fluid Mechanics, 36(1):381-411, January 2004. doi:10.1146/annurev.fluid.36.050802.122124 C838

R.-J. Yang, L.-M. Fu, and C.-C. Hwang. Electroosmotic entry flow in a microchannel. Journal of Colloid and Interface Science, 244:173-179, 2001. doi:10.1006/jcis.2001.7847 C838

R.-J. Yang, T.-I. Tseng, and C.-C Chang. End effects on electro-osmotic flows in micro-channels. Journal of Micromechanics and Microengineering, 15:254-262, 2005. doi:10.1088/0960-1317/15/2/002 C838

\section{Author addresses}

1. J. D. Berry, Dept of Chemical and Biomolecular Engineering, The University of Melbourne, Parkville, Victoria 3010, Australia. mailto:joe.d.berry@gmail.com

2. M. R. Davidson, Dept of Chemical and Biomolecular Engineering, The University of Melbourne, Parkville, Victoria 3010, Australia.

3. D. J. E. Harvie, Dept of Chemical and Biomolecular Engineering, The University of Melbourne, Parkville, Victoria 3010, Australia. 